\title{
Behavior of Neutral Particles under Charge Conjugation
}

\author{
M. Geld-Mann,* Department of Physics, Columbia University, New York, New York
}

AND

\author{
A. PaIs, Institute for Advanced Study, Princeton, New Jersey
}

(Received November 1, 1954)

\begin{abstract}
Some properties are discussed of the $\theta^{0}$, a heavy boson that is known to decay by the process $\theta^{0} \rightarrow \pi^{+}+\pi^{-}$. According to certain schemes proposed for the interpretation of hyperons and $K$ particles, the $\theta^{0}$ possesses an antiparticle $\bar{\theta}^{0}$ distinct from itself. Some theoretical implications of this situation are discussed with special reference to charge conjugation invariance. The application of such invariance in familiar instances is surveyed in Sec. I. It is then shown in Sec. II that, within the framework of the tentative schemes under consideration, the $\theta^{0}$ must be considered as a "particle mixture" exhibiting two distinct lifetimes, that each lifetime is associated with a different set of decay modes, and that no more than half of all $\theta^{0}$ 's undergo the familiar decay into two pions. Some experimental consequences of this picture are mentioned.
\end{abstract}

\section{I}

$I^{T}$ $\mathrm{T}$ is generally accepted that the microscopic laws of physics are invariant to the operation of charge conjugation (CC); we shall take the rigorous validity of this postulate for granted. Under CC, every particle is carried into what we shall call its "antiparticle". The principle of invariance under $\mathrm{CC}$ implies, among other things, that a particle and its antiparticle must have exactly the same mass and intrinsic spin and must have equal and opposite electric and magnetic moments.

A charged particle is thus carried into one of opposite charge. For example, the electron and positron are each other's antiparticles; the $\pi^{+}$and $\pi^{-}$and the $\mu^{+}$ and $\mu^{-}$mesons are supposed to be pairs of antiparticles; and the proton must possess an antiparticle, the "antiproton".

Neutral particles fall into two classes, according to their behavior under CC:

(a) Particles that transform into themselves, and which are thus their own antiparticles. For instance the photon and the $\pi^{0}$ meson are bosons that behave in this fashion. It is conceivable that fermions, too, may belong to this class. An example is provided by the Majorana theory of the neutrino.

In a field theory, particles of class (a) are represented by "real" fields, i.e., Hermitian field operators. There is an important distinction to be made within this class, according to whether the field takes on a plus or a minus sign under CC. The operation of CC is performed by a unitary operator $\mathrm{C}$. The photon field operator $A_{\mu}(x)$ satisfies the relation

$$
\mathcal{C} A_{\mu}(x) \mathfrak{C}^{-1}=-A_{\mu}(x)
$$

while for the $\pi^{0}$ field operator $\phi(x)$ we have

$$
\mathfrak{e} \phi(x) \mathfrak{C}^{-1}=\phi(x) .
$$

Equation (1) expresses the obvious fact that the electromagnetic field changes sign when positive and negative charges are interchanged; that the $\pi^{0}$ field

* On leave from Department of Physics and Institute for Nuclear Studies, University of Chicago, Chicago, Illinois. must not change sign can be inferred from the observed two-photon decay of the $\pi^{0}$.

We are effectively dealing here with the "charge conjugation quantum number" $C$, which is the eigenvalue of the operator $\mathfrak{e}$, and which is rigorously conserved in the absence of external fields. If only an odd (even) number of photons is present, we have $C=-1(+1)$; if only $\pi^{0}$ s are present, $C=+1$; etc. As a trivial example of the conservation of $C$, we may mention that the decay of the $\pi^{0}$ into an odd number of photons is forbidden. ${ }^{1}$

We may recall that a state of a neutral system composed of charged particles may be one with a definite value of $C$. For example, the ${ }^{1} S_{0}$ state of positronium has $C=+1$; a state of a $\pi^{+}$and a $\pi^{-}$ meson with relative orbital angular momentum $l$ has $C=(-1)^{l}$; etc.

For fermions, as for bosons, a distinction may be made between "odd" and "even" behavior of neutral fields of class (a) under CC. However, the distinction is then necessarily a relative rather than an absolute one. ${ }^{2}$ In other words, it makes no sense to say that a single such fermion field is "odd" or "even", but it does make sense to say that two such fermion fields have the same behavior under CC or that they have opposite behavior.

(b) Neutral particles that behave like charged ones in that: (1) they have antiparticles distinct from themselves; (2) there exists a rigorous conservation law that prohibits virtual transitions between particle and antiparticle states.

A well-known member of this class is the neutron $N$, which can obviously be distinguished from the antineutron $\bar{N}$ by the sign of its magnetic moment. The law that forbids the virtual processes $N \rightleftarrows \bar{N}$ is the law

1 For other consequences of invariance under charge conjugation see A. Pais and R. Jost, Phys. Rev. 87, 871 (1952); L. Wolfenstein and D. G. Ravenhall, Phys. Rev. 88, 279 (1952); L. Michel, Nuovo cimento 10, 319 (1953).

${ }^{2}$ This is due to the fact that fermion fields can interact only bilinearly. For example, one easily sees that the interactions responsible for $P \rightarrow N+e^{+}+\nu$ would not lead to physically distinguishable results if $\nu$ were either an even or an odd Majorana neutrino. 
of conservation of baryons, ${ }^{3}$ which is, so far as we know, exact, and which states that $n$, the number of baryons minus the number of antibaryons, must remain unchanged. Clearly all neutral hyperons likewise belong to this class. Although we know of no "elementary" bosons in the same category, we have no a priori reason for excluding their existence. [Note that the $\mathrm{H}$ atom is an example of a "non-elementary" boson of class (b).]

Particles in this class are represented by "complex" fields, and the operation of charge conjugation transforms the field operators into their Hermitian conjugates.

It is the purpose of this note to discuss the possible existence of neutral particles that seem, at first sight, to belong neither to class (a) nor to class (b).

\section{II}

Recently, attempts have been made to interpret hyperon and $K$-particle phenomena by distinguishing sharply between strong interactions, to which essentially all production of these particles is attributed, and weak interactions, which are supposed to induce their decay. It is necessary to assume that the strong interactions give rise to "associated production "exclusively."

Certain detailed schemes ${ }^{5}$ which meet this requirement lead to further specific properties of particles and interactions. In particular, a suggestion has been made about the $\theta^{0}$ particle, a heavy boson that is known to decay according to the scheme:

$$
\theta^{0} \rightarrow \pi^{+}+\pi^{-}+(\sim 215 \mathrm{Mev}) .
$$

It has been proposed that the $\theta^{0}$ possesses an antiparticle $\vec{\theta}^{0}$ distinct from itself, and that in the absence of the weak decay interactions, there is a conservation law that prohibits the virtual transitions $\theta^{0} \rightleftarrows \bar{\theta}^{0}$. [In our present language, we would say that the $\theta^{0}$ belongs to class (b) if the weak interactions are turned off.] This conservation law also leads to stability of the $\theta^{0}$ and $\bar{\theta}^{0}$; moreover, while it permits the reaction $\pi^{-}+P \rightarrow \Lambda^{0}+\theta^{0}$ it forbids the analogous process $\pi^{-}+P \rightarrow \Lambda^{0}+\bar{\theta}^{0}$. In the schemes under consideration this is the same law that forbids the reaction: 2 neutrons $\rightarrow 2 \Lambda^{0}$.

The weak interactions that must be invoked to account for the observed decay (3) evidently cause the conservation law to break down (a fact that is, of course, of little importance for production). This breakdown makes the forbiddenness of the processes $\theta^{0} \rightleftarrows \bar{\theta}^{0}$ no longer absolute, as can be seen from the following argument: In the decay (3) the pions are left in a state with a definite relative angular momentum and therefore with a definite value of the chargeconjugation quantum number $C$. The charge-conjugate process,

$$
\bar{\theta}^{0} \rightarrow \pi^{+}+\pi^{-},
$$

${ }^{3}$ Nucleons and hyperons are collectively referred to as baryons.

4 A. Pais, Phys. Rev. 86, 663 (1952).

${ }^{5}$ M. Gell-Mann, Phys. Rev. 92, 833 (1953); A. Pais, Proc. Nat, Acad. Sci. U. S. 40, 484, 835 (1954); M. Gell-Mann and A. Pais. Proceedings of the International Conference Glasgow (Pergamon Press, London, to be published). must also occur and must leave the pions in the same state; moreover the reverse of (4) must also be possible, at least as a virtual process. Therefore the virtual transition $\theta^{0} \rightleftarrows \pi^{+}+\pi \rightleftarrows \bar{\theta}^{0}$ is induced by the weak interactions, and we are no longer dealing exactly with case (b).

In order to treat this novel situation, we shall find it convenient to introduce a change of representation. Since the $\theta^{0}$ and $\bar{\theta}^{0}$ are distinct, they are associated, in a field theory, with a "complex" field $\psi$ (a nonHermitian field operator), just as in case (b). Under charge conjugation $\psi$ must transform according to the law:

$$
\begin{aligned}
& \mathfrak{e} \psi \mathfrak{C}^{-1}=\psi^{+} \\
& \mathfrak{e} \psi^{+} \mathfrak{e}^{-1}=\psi
\end{aligned}
$$

where $\psi^{+}$is the Hermitian conjugate of $\psi$. Let us now define

$$
\begin{aligned}
& \psi_{1} \equiv\left(\psi+\psi^{+}\right) / \sqrt{2} \\
& \psi_{2} \equiv\left(\psi-\psi^{+}\right) / \sqrt{2} i
\end{aligned}
$$

so that $\psi_{1}$ and $\psi_{2}$ are Hermitian field operators satisfying

and

$$
\mathfrak{C} \psi_{1} \mathfrak{e}^{-1}=\psi_{1}
$$

$$
\mathfrak{e} \psi_{2} \mathfrak{e}^{-1}=-\psi_{2} \text {. }
$$

The fields $\psi_{1}$ and $\psi_{2}$ evidently correspond to class (a); in fact $\psi_{1}$ is "even" like the $\pi^{0}$ field and $\psi_{2}$ is "odd" like the photon field. Corresponding to these real fields there are quanta, which we shall call $\theta_{1}{ }^{0}$ and $\theta_{2}{ }^{0}$ quanta. The relationship that these have to the quanta of the complex $\psi$ field, which we have called $\theta^{0}$ and $\bar{\theta}^{0}$, may be seen from an example: Let $\Psi_{1}$ be the wave-functional representing a single $\theta_{1}$ quantum in a given state, while $\Psi_{0}$ and $\Psi_{0}^{\prime}$ describe a $\theta^{0}$ and a $\bar{\theta}^{0}$, respectively, in the same state. Then we have

$$
\Psi_{1}=\left(\Psi_{0}+\Psi_{0}^{\prime}\right) / \sqrt{2} \text {. }
$$

Thus the creation of a $\theta_{1}$ (or, for that matter, of a $\theta_{2}$ ) corresponds physically to the creation, with equal probability and with prescribed relative phase, of either a $\theta^{0}$ or a $\vec{\theta}^{0}$. Conversely, the creation of a $\theta^{0}$ (or of a $\vec{\theta}^{0}$ ) corresponds to the creation, with equal probability and prescribed relative phase, of either a $\theta_{1}{ }^{0}$ or a $\theta_{2}{ }^{0}$.

The transformation (6), (7) to two real fields could equally well have been applied to a complex field of class (b), such as that associated with the neutron. However, this would not be particularly enlightening. It would lead us, for instance, to describe phenomena involving neutrons and antineutrons in terms of " $N_{1}$ and $N_{2}$ quanta". Now a state with an $N_{1}$ (or $N_{2}$ ) quantum is a mixture of states with different values of the quantum number $n$, the number of baryons minus the number of antibaryons. But the law of conservation of baryons requires this quantity to be a constant of the motion, and so a mixed state can never arise from a pure one. Since in our experience we deal exclusively 
with states that are pure with respect to $n$, the introduction of $N_{1}$ and $N_{2}$ quanta can only be a mathematical device that distracts our attention from the truly physical particles $N$ and $\bar{N}$.

On the other hand, it can obviously not be argued in a similar way that the $\theta_{1}{ }^{0}$ and $\theta_{2}{ }^{0}$ quanta are completely unphysical, for the corresponding conservation law in that case is not a rigorous one. Always assuming the correctness of our model of the $\theta^{0}$, we still have the $\theta^{0}$ and $\bar{\theta}^{0}$ as the primary objects in production phenomena. But we shall now show that the decay process is best described in terms of $\theta_{1}{ }^{0}$ and $\theta_{2}{ }^{0}$.

The weak interactions, in fact, must lead to very different patterns of decay for the $\theta_{1}{ }^{0}$ and $\theta_{2}{ }^{0}$ into pions and (perhaps) $\gamma$ rays; any state of pions and/or $\gamma$ rays that is a possible decay mode for the $\theta_{1}{ }^{0}$ is not a possible one for the $\theta_{2}{ }^{0}$, and vice versa. This is because, according to the postulate of rigorous $\mathrm{CC}$ invariance, the quantum number $C$ is conserved in the decay; the $\theta_{1}{ }^{0}$ must go into a state that is even under charge conjugation, while the $\theta_{2}{ }^{0}$ must go into one that is odd. Since the decay modes are different and even mutually exclusive for the $\theta_{1}{ }^{0}$ and $\theta_{2}{ }^{0}$, their rates of decay must be quite unrelated. There are thus two independent lifetimes, one for the $\theta_{1}{ }^{0}$, and one for the $\theta_{2}{ }^{0}$.

An important illustration of the difference in decay modes of the $\theta_{1}{ }^{0}$ and $\theta_{2}{ }^{0}$ is provided by the two-pion disintegration. We know that reaction (3) occurs; therefore at least one of the two quanta $\theta_{1}{ }^{0}$ and $\theta_{2}{ }^{0}$, say $\theta_{1}{ }^{0}$, must be capable of decay into two charged pions. The final state of the two pions in the $\theta_{1}{ }^{0}$ decay is then even under charge conjugation like the $\theta_{1}{ }^{0}$ state itself. These two pions are thus in a state of even relative angular momentum and therefore of even parity. So the $\theta_{1}{ }^{0}$ must have even spin and even parity. Now we assume that the $\theta^{0}$ has a definite intrinsic parity, and therefore the parity and spin of the $\theta_{2}{ }^{0}$ must be the same as those of the $\theta_{1}{ }^{0}$, both even. If the $\theta_{2}{ }^{0}$ were to decay into two pions, these would again be in a state of even relative angular momentum and thus even with respect to charge conjugation. However, the $\theta_{2}{ }^{0}$ is itself odd under charge conjugation; its decay into two pions is therefore forbidden.

Alternatively, if the $\theta_{2}{ }^{0}$ is the one that actually goes into two pions, then the spin and parity of $\theta_{1}{ }^{0}$ and the $\theta_{2}{ }^{0}$ are both odd, and so the $\theta_{1}{ }^{0}$ cannot decay into two pions.

Of the $\theta_{1}{ }^{0}$ and the $\theta_{2}{ }^{0}$, that one for which the two-pion decay is forbidden may go instead into $\pi^{+}+\pi^{-}+\gamma$ or possibly into three pions (unless the spin and parity of the $\theta^{0}$ are $0^{+}$), etc.

While we have seen that the $\theta_{1}{ }^{0}$ and $\theta_{2}{ }^{0}$ may each be assigned a lifetime, this is evidently not true of the $\theta^{0}$. or $\bar{\theta}^{0}$. Since we should properly reserve the word "particle" for an object with a unique lifetime, it is the $\theta_{1}{ }^{0}$ and $\theta_{2}{ }^{0}$ quanta that are the true "particles". The $\theta^{0}$ and the $\bar{\theta}^{0}$ must, strictly speaking, be considered as "particle mixtures."
It should be remarked that the $\theta_{1}{ }^{0}$ and the $\theta_{2}{ }^{0}$ differ not only in lifetime but also in mass, though the mass difference is surely tiny. The weak interactions responsible for decay cause the $\theta_{1}{ }^{0}$ and the $\theta_{2}{ }^{0}$ to have their respective small level widths and correspondingly must produce small level shifts which are different for the two particles.

To sum up, our picture of the $\theta^{0}$ implies that it is a particle mixture exhibiting two distinct lifetimes, that each lifetime is associated with a different set of decay modes, and that not more than half of all $\theta^{0}$ 's can undergo the familiar decay into two pions. ${ }^{6}$

We know experimentally that the lifetime $\tau$ for the decay mode (3) (and hence for all decay modes that may compete with this one) is about $1.5 \times 10^{-10} \mathrm{sec}$. The present qualitative considerations, even if at all correct in their underlying assumptions, do not enable us to predict the value of the "second lifetime" $\tau^{\prime}$ of the $\theta^{0}$. Nevertheless, the examples given above of decays responsible for the second lifetime lead one to suspect that $\tau^{\prime} \gg \tau$. As an illustration of the experimental implications of this situation consider the study of the reaction $\pi^{-}+P \rightarrow \Lambda^{0}+\theta^{0}$ in a cloud chamber. If the reaction occurs and subsequently $\Lambda^{0} \rightarrow P+\pi^{-}$, $\theta^{0} \rightarrow \pi^{+}+\pi^{-}$, there should be a reasonable chance to observe this whole course of events in the chamber, as the lifetime for the $\Lambda^{0}$ decay $\left(\sim 3.5 \times 10^{-10} \mathrm{sec}\right)$ is comparable to $\tau$. However, if it is true that $\tau^{\prime} \gg \tau$, it would be very difficult to detect the decay with the second lifetime in the cloud chamber with its characteristic bias for a limited region of lifetime values. ${ }^{8}$ Clearly this also means an additional complication in the determination from cloud chamber data as to whether or not production always occurs in an associated fashion. In some such cases the analysis of the reaction $\pi^{-}+P \rightarrow \Lambda^{0}+$ ? may still be pushed further, however, if one assumes that besides the $\Lambda^{0}$ only one other neutral object is formed. ${ }^{9}$

At any rate, the point to be emphasized is this: a neutral boson may exist which has the characteristic $\theta^{0}$ mass but a lifetime $\neq \tau$ and which may find its natural place in the present picture as the second component of the $\theta^{0}$ mixture.

One of us, (M. G.-M.), wishes to thank Professor E. Fermi for a stimulating discussion.

\footnotetext{
${ }^{6}$ Note that if the spin and parity of the $\theta^{0}$ are even, then the $\theta_{1}{ }^{0}$ may decay into $2 \pi^{0} \mathrm{~s}$ as well as into $\pi^{+}+\pi^{-}$.

${ }^{7}$ The process $\theta^{0} \rightarrow \pi^{+}+\pi^{-}+\gamma$ may occur as a radiative correction to the allowed decay into $\pi^{+}+\pi^{-}$connected with the lifetime $\tau$; see S. B. Treiman, Phys. Rev. 95, 1360 (1954). The process may also occur as one of the principal decay modes associated with the second lifetime $\tau^{\prime}$. The latter case may be distinguished from the former not only by the distinct lifetime but also by a different energy spectrum which probably favors higher $\gamma$-ray energies; such a spectrum is to be expected in a case where the emission of the $\gamma$ ray is not just part of the "infrared catastrophe", but is an integral part of the decay process.

${ }^{8}$ See, e.g., Leighton, Wanlass, and Anderson, Phys. Rev. 89, 148 (1953), Sec. III

${ }^{9}$ See Fowler, Shutt, Thorndike, and Whittemore, Phys. Rev. 91, 1287 (1953).
} 